\title{
A Study on Computer-aided Translation Technology in the Era of Globalization and Information
}

\author{
Xiang LI \\ School of Foreign Studies \\ North China University of Water Resources and Electric Power \\ Zhengzhou, 450046 China
}

\begin{abstract}
In the era of globalization, the rapid development of modern information technology, which greatly enhances the productivity of the translation, has an unprecedented impact on the language service industry. Based on this background, this paper describes the basic concepts of computer-aided translation technologies, expounds the important role of major computer-aided translation technology in modern translation practice, and analyzes the development trend of computeraided translation technologies. Finally, the article points out computer-aided translation technology has become an integral part of modern translation studies, which has far-reaching significance for improving language service industry, and promoting innovation translation theory.
\end{abstract}

Keywords- computer-aided translation; machine translation, translation memory

\section{INTRODUCTION}

With the rapid advancement of international political, economic, technological and cultural exchanges in the era of globalization and information, globalized translation has been playing an increasingly large role, and in the meanwhile cultural differences between different ethnic groups continue to shrink. Globalized translation requires source language cultures and target language cultures to keep open with each other and talk to each other. However, in fact, the cultural collisions in translation exist throughout the entire process of translation. The revolution of information technology comprising computers, networking and communication technology has penetrated into all areas of the society. The power of technology has affected all aspects of social life. Linguists and natural language processing experts have long been committed to the pursuit of artificial intelligence, allowing fully automated computer translation technology to solve the ever-growing problems of language translation and communication. Therefore, computer-aided translation (CAT) techniques and tools have emerged. The concept of computer-assisted translation has been discussed by many domestic and foreign scholars. To sum up, CAT can be divided into two types, on in a narrow sense and one in a broad sense. Computer-aided translation technology in a narrow sense usually refers to the translation technology that applies translation memory matching technology to improve translation efficiency, which uses a computer to simulate the mechanisms of human brain memory function, leaving the simple and repetitive memory activities in the translation process to the computer, and liberating translators from mechanical work so that they can fully focus on the translation itself. Computer-aided translation is a translation activity carried out mainly by man, which is distinguished from fully automated machine translation. The former can be called "human translation aided by machine" while the latter can be called "machine translation aided by human". Computer-aided translation technology in a broad sense is not limited to the above and it may involve information technology that the translator may use to improve the translation efficiency, for example, before translation: the encoding processing, extraction of translation resource, word count, task analysis, extraction of terminology and so on; during translation: reuse of translated fragments, searching, verification, term identification and progress monitoring; after translation: format conversion, fuzzy matching, automated quality assurance, language asset management, etc; and corpus automatic alignment, machine translation, voice input, voice translation and other technologies.

\section{INTRODUCTION TO COMPUTER-AIDED TRANSLATION TECHNOLOGY}

Machine Translation (MT) was born with the advent of the electronic age. Although people doubted the development and prospects of machine translation, now computer translation technology has made great progress through researchers' continuous efforts and innovation. The Montreal University Canada and the Translation Bureau of Canadian Federal Government jointly developed TAUMMETEO system, a large practical machine translation system, which is mainly used for the translation of weather forecasting literature. The TAUM-METEO system is capable of English-French translation, 60,000 to 300,000 words per hour, 1,500 to 2,000 passages of weather forecast information a day. In addition, the SYSTRAN machine translation system is also a very successful system, which is most widely used in Europe and America. The SYSTRAN system can achieve translation between English and other European languages. The Overseas Technical Department of the American Air Force, which translates more than 100,000 pages of intelligence information with SYSTRAN system every year, reported that the majority of the translations for browsing can be used without editing and only about $20 \%$ of 
the translation shall be slightly processed before delivery, with translation accuracy rate of $90-95 \%$.

Back in 1984, Japan began using MEDIUM-PAK to carry out Japanese-English translation of scientific texts and articles in newspapers and magazines and has developed ATLAS and HICATS systems. Among our machine translation systems are "Yixing No.1" by CS\&S, "Gaoli" System in Beijing, "863 English-Chinese Smart Machine Translation System", "Langway" system in Shanxi, "Tongyi" system in Tianjin, "Sunshine Yiwang" system in Guangdong, and other translation systems produced in Hong Kong and Taiwan. However, due to the limitations of computer technology itself and the level of human knowledge, both machine translation aided by human and human translation aided by machine have now met with some temporary insurmountable obstacles. The developed computer software can only meet the needs of translation for part fields, certain occasions and some literary forms. Therefore, people turn to search for new solutions to the problems based on full understanding of the strengths and limitations of the computers. With the emergence and development of the Internet, the constant updates of computer hardware and software, and the expansion of computer storage capacity, now the machine translation systems have paid more and more attention to the construction of corpuses. Some research institutions are trying an integrated translation approach, using statistical methods and focusing on the combination of corpuses. One of the achievements is Translation Memory (TM).

\section{ADVANTAGES OF COMPUTER-AIDED TRANSLATION TECHNOLOGY}

Traditional manual translation cannot satisfy the market where more and more translation is demanded. However, in comparison, computer-aided translation has many notable advantages. Computer-aided translation based on Translation Memory facilitates translation and reduces time with the help of Quality Assurance tools, Translation Management System, Translation Servers and other technologies.

\section{A. Saving Time and Manpower}

Translation memory is the most significant technological achievement of computer-aided translation development. During the translation process, computer-aided translation software will store the original text and its translation into the language database, i.e. the translation memory. When repetitive or highly similar phrases, sentences and chapters appear, the system will search the translation memory and then automatically prompt the user to recycle the closest translation based on computer-aided matching technology. Provided with the appropriate reference, the translator can adopt, edit, or give up the reference translation as necessary. When similar text is being translated next time, the system will automatically pre-translate the text for the translator, who will translate only the remaining. Therefore, with the help of computer-aided translation technology, reuse of existing translation may save translation time, avoiding duplication of work, greatly enhancing the efficiency and speed of translation.

\section{B. Collaborating to Achieve Translation}

Traditionally, one translation task is conducted by one translator in order to keep translation accordant in wording style. However, at present, one translator cannot conduct one task solely on his own because of huge translation amount and time limit. But cooperative translation makes it difficult to keep language style uniform. Computer-aided translation provides a solution to this problem. Translators can communicate with each other during translation. Within the specified time, under conditions have sufficient human resources, if you want to translate the huge amount of quality text, you must rely on computer-assisted translation technologies to give advanced scientific analysis to the behavior of the entire translation implementation. Collaborative translation drawbacks traditional artificial selfevident, its performance is very difficult to quantify the process of translation, the translation of the term is difficult to achieve unity, cannot monitor the quality of translation, translation standard is difficult to regulate, making the integration of translation collaboration translations consumption time-consuming, and ultimately affect the timeliness and quality of the translation project. Translation Memory technology for translation project management and distribution helps more translator to translate the term storebased cooperative management, to achieve more term harmonization of standards and norms translation, so that translation collaboration achieve the desired effect. In the computer-assisted translation cooperative mode, assisted translation software is set to provide an interpreter for over shared memory, different translators' translation style coordination, and strengthen exchanges and cooperation in sharing resources among translators to ensure a unified and standardized terminology, for efficient translation project management and collaboration.

\section{APPLICATION OF COMPUTER-AIDED TRANSLATION TECHNOLOGY}

Translation Memory Technologies. Translation Memory stores the translated texts and the original ones together and achieves search and information extraction of the unstructured documents by adding labels to the documents. When using CAT tools for translation, the system will cut the new text into fragments, retrieve the translation memory, match them with the original texts stored in the database, and replace the new texts with the translations of the same or similar fragments. Translation memory applications reduce duplication of translators' work and improve translation efficiency. However, there are limits to the translation memory.

Translation Server. In order to solve the problem of unification which happens when multi-translators are involved in one translation project, in addition to extracting terminology and high repetition rate of segments before the translation, an important solution is the real-time sharing memories and glossaries. Because the terminology extraction technology is not perfect at this stage, some terms, especially multi-word terms, cannot be effectively identified in many cases. Therefore, in the process of translation, translators 
often add those terms to the database after encountering them. In order to ensure consistency of translation, we can build a translation server so that translators can use real-time updated translation memory.

Revision. In small translation projects, revision work is generally done by translators. However, the scale of modern translation projects is increasing and an independent reviser is appointed to undertake the revision. A reviser's workload is no less than a translator as he is often responsible for a number of translators. Therefore, revisers need to use computer-aided system to improve work efficiency. A quality assurance tool is such software. Some CAT software contains revision components. QA tools are able to detect basic mistakes in the translation, including omission, term errors, inconsistent translation, format mistakes and other default errors. Some QA tools allow users to define their own error types and search for them in the translation automatically.

Other Technical Applications. With the development of technology, translation has developed from simple bilingual translation to an integrative field which involves project management, publishing, printing and so on. In addition to computer assisted translation tools, a translation project uses a translation project management software, text and image processing software, etc. Optical Character Recognition software is one of the software commonly used in the translation industry. Although the proportion of modern electronic documents is gradually increasing, the traditional paper-based documents still occupy a certain proportion, which need to be transformed electronically for computeraided translation. In addition to manual input, scanners are commonly used to obtain picture files, which are then put into machine-readable files through OCR software.

\section{DEVELOPMENT TRENDS}

As a substitute for machine translation, the goal of computer-aided translation technology is to improve the level of automation and change the present computer-aided translation into computer translation in order to save manpower and improve production efficiency. The current mainstream software CAT technology is relatively simple, with a low level of automation. If the language model in the field of natural language processing can be introduced and the system is further optimized, the efficiency of the system can be greatly improved. Based on the practice of computeraided translation, the level of automation can be increased. First, the producing and retrieval of translation memory will be more convenient and efficient. The production of TM through aligning is the common work of computer-aided translation. A lot of software has aligning module for TM producing, but the aligning quality is different. TRADOS's alignment tool WinAlign needs manual proof and much correction although the effect is relatively ideal. So, it can only be regarded as a semi-automated tool. Corpus aligning is necessary in natural language processing. The aligning algorithm based on length was put forward in 1991, which makes alignment according to the number of words in each sentence and greatly improves the quality of alignment, with alignment accuracy rate of $90 \%$. This aligning method assumes that the original long sentences will be translated into long sentences and the original short sentences will be translated into short sentences. But since there may be one-to many, many-to-many and many-to-one relationships in the bilingual conversions, it is necessary to calculate the approximate percentage and identify specific correspondence before aligning. Thus, the introduction of aligning method based on the length can improve the accuracy. During translation, you need to retrieve translation memories to find the right translation. There are two kinds of search results, exact matching and fuzzy matching. Exact matching returns to the segment in the memory that is $100 \%$ the same as the new text; fuzzy matching returns to similar fragment.

\section{CONCLUSIONS}

The exchange and integration of domestic and foreign markets are being accelerated and the international exchanges and cooperation are ever-increasing, which offers unprecedented good opportunities for the rapid development of the translation market. While more and more foreign companies set their sights on the Chinese mainland, a sharp requirement for information localization is increasing. The development of the translation market has put forward higher demand for personnel training. In the professional translation field, computer-aided translation technology is being more widely used.

\section{References}

[1] Zhu Guicheng. Cultural Globalization $\bullet$ Cultural Integrity $\bullet$ Translation Strategies [J] Journal of Hehai University (Philosophy and Social Sciences), 2002,03: 74-76.

[2] Zhao Shuming. Translator's Cultural Identity in Globalized Translation [J] Journal of Mudanjiang Teachers College (Philosophy and Social Sciences), 2002,02: 61-63.

[3] Wang Huashu. A Study on the Computer-aided Translation Technologies in the Information Age [J] Foreign Studie, 2014,03: 92-97 + 108.

[4] Shan Yu. The Application of Computer-aided Translation Technology in the Practical English Teaching [J] Vocational and Technical Education, 2013,23: 48-49.

[5] Liu Si. On the Advantages and Disadvantages of Computer-aided Translation Technology [J]. Journal of Chongqing College of Electronic Engineering, 2014, 06: 89-91.

[6] Jin Guangsa. Of Computer-aided Translation Technology Status and Development Trend Dynasty [J] Shenyang Institute of Engineering (Natural Science), 2010,03: 264-266 + 280 . 